\title{
Malignant solitary fibrous tumor of urinary bladder: A rare clinical entity
}

\author{
Zisis Kratiras ${ }^{1,2,3}$, Vasileios Spapis ${ }^{1}$, Efthymios Koniaris ${ }^{2}$, Diomidis Kozyrakis ${ }^{3}$, \\ Konstantinos Skriapas ${ }^{4}$ \\ ${ }^{1}$ Department of Urology, Hippokratio General Hospital of Athens, Athens, Greece; \\ ${ }^{2}$ Department of Pathology, Hippokratio General Hospital of Athens, Athens, Greece; \\ ${ }^{3}$ Department of Urology, General Hospital of Volos, Volos, Greece; \\ ${ }^{4}$ Department of Urology, General Hospital of Larisa, Larisa, Greece.
}

\begin{abstract}
Summary Solitary Fibrous Tumors (SFTs) are mesenchymal tumors occurring in several sites. Urinary bladder SFTs are quite rare. Eighteen cases are described in the literature and only two of them had malignant features. SFTs comprise a histologic spectrum of mesenchymal neoplasms that show fibroblastic differentiation. The signs and symptoms are non specific. Immunohistochemistry plays a pivotal role in the diagnosis, differentiating SFTs from other spindle cell mesenchymal tumors. Malignant criteria are considered the large size, increased mitotic activity, focal necrosis or hemorrhage, nuclear atypia, hypercellularity and infiltrative margins. Clinical and biological behavior of bladder SFTs is usually not aggressive but cannot be safely predicted based on the pathologic features. Complete surgical resection is the cornerstone of treatment. We present the third bladder SFT case with malignant features and a mini literature review.
\end{abstract}

KEY WORDS: Solitary fibrous tumor; Hemangiopericytoma; Bladder tumors; Mesenchymal tumors.

Submitted 17 February 2019; Accepted 11 March 2019

\section{INTRODUCTION:}

In 1931 Klemperer and Rabin were the first to describe the solitary fibrous tumor (SFT) as a mesothelial tumor arising from the pleura. Since then numerous extrapleural sites of SFT had been described (1). SFTs originating from the urinary bladder are uncommon; only 18 cases have been described in the English literature. We report a case of a SNT of the bladder with malignant features in a 31-year old male patient.

\section{Case report}

A 31-year old male patient self-presented to the Accident and Emergency department of our hospital reporting frank heamaturia, accompanied with dysuria, dull abdominal pain and blood clots. He denied any systemic symptoms and his clinical examination was unremarkable. From his past medical history, he was an ex-smoker but otherwise fit and healthy. His blood chemistry was in the normal range. A computed tomography (CT) that was performed revealed a $42 \times 53 \mathrm{~mm}$ solid mass at the left lateral wall and the bladder dome without any evidence of lymph nodes or distant metastasis. Patient was driven to theatre and an urgent transurethral resection was performed, revealing a solid looking bladder tumor occupying the bladder dome and part of the left lateral wall.

The pathology revealed that the tumor was composed of ovoid- to spindle-shaped cells with alternating hypercellular and hypocellular areas. They had a staghorn vascular pattern with a mixed variable with evidence of necrosis and hemorrhage. Nuclear atypia was present with increased mitotic activity, focally up to 23/10 HPF, and a proliferation rate Ki67 positive 30-35\% (Figure 1).

Immunohistochemistry revealed that the tumor was positive for Vimentin, bcl-2, CD99 and CD34 and negative for Actin, Desmin, CD117 and CKAEl/AE3. Patient underwent a magnetic resonance imaging (MRI) for local staging followed by an uneventful radical cystectomy and ileal conduit as diversion. The tumor was completely removed with clear surgical margins. He had a successful recovery

Figure 1.

Pathology revealed ovoid- to spindle-shaped cells with a staghorn vascular pattern.

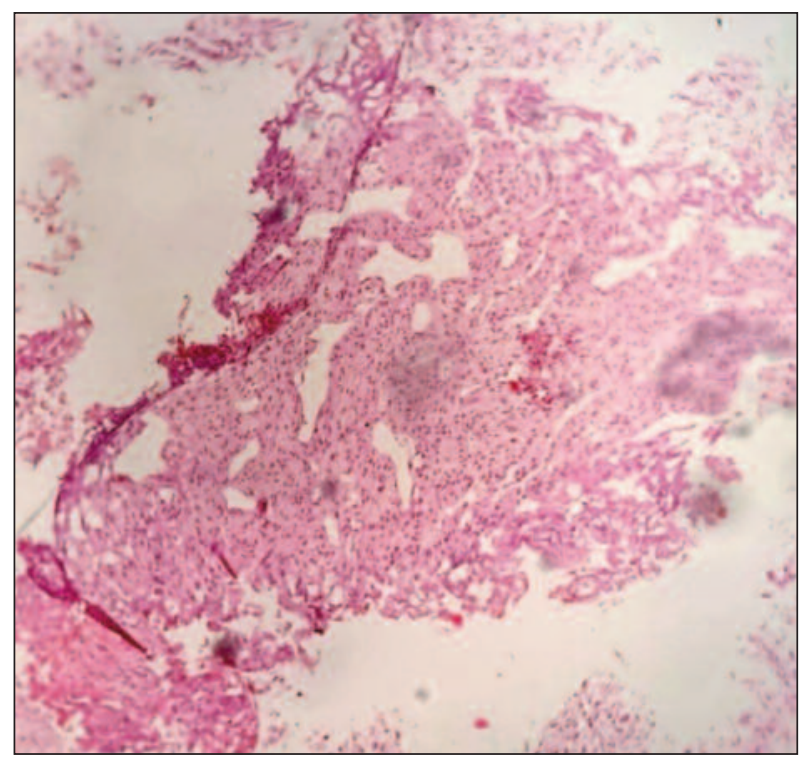

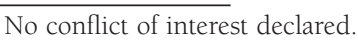


without any immediate post operative complication. He was discharged home at day 9 post op and he was reviewed in clinic after 3 months. His final pathology verified the initial diagnosis of SNT of the bladder with malignant features.

\section{Discussion}

Solitary fibrous tumors comprise a histologic spectrum of rarely metastasizing mesenchymal neoplasms that show fibroblastic differentiation and can occur in any anatomical position. Extrapleural SFTs are usually diagnosed between the $5^{\text {th }}$ and the 6th decade of life, having an equal distribution between races and sexes. They are normally slowly growing and symptoms arise due to local invasion of the tumor. The usual presenting symptoms are abdominal pain along with palpable abdominal mass. Heamaturia and dysuria might also be the first symptoms of a bladder SFT as in our case. Symptomatic hypoglycemia might occur as paraneoplasmatic syndrome in a small subset of tumors (2).

There is no diagnostic or treatment algorithm. Imaging plays the most contemporary role in the diagnosis. Certain, but not specific, imaging features have been described. CT usually reveals an enhancing heterogenous, well defined mass demonstrating the vascular nature of the tumor. Areas of hemorrhage or necrosis might be present. MRI usually reveals heterogenous and variable signals depending on the vascularity and the fibrous stroma of the neoplasm.

Macroscopically the tumor is usually a well circumscribed, tan colored mass that may be encapsulated by a thin and vascular pseudocapsule. It usually arises from the bladder submucosa although cases arising from bladder serosa have been described. Microscopically it consists of hypocelullar and hypercellular areas consisting of ovoid and spindle-shaped cells (2, 3). Staghorn configuration of blood vessels is common.

Immunohistochemistry plays a pivotal role in differentiating SFTs from other spindle cell mesenchymal tumors. SFTs are immunoreactive to Vimentin, CD34, CD99 and bcl-2 and they are negative for actin, desmin (in smooth muscle tumors), keratin and CD117 (in GISTs) $(2,3)$.

Malignant criteria are considered the large size, increased mitotic activity (more than 4 mitosis in 10 high power fields), focal necrosis or hemorrhage, nuclear atypia, hypercellularity and infiltrative margins. Clinical and biological behavior of bladder SFTs is usually not aggressive but cannot be safely predicted based on the pathologic features. Even benign tumors can act aggressively by invading structures at the proximity of the tumor or reoccurring, while malignant ones might have a less aggressive behavior without recurrence or metastasis $(2,3)$.

Complete surgical resection is the cornerstone of treatment. Adjuvant radiotherapy is a useful asset for the local control of the disease, while adjuvant chemotherapy appears to have limited efficacy. Chemoradiotherapy has been used for non-resectable tumors with variable success. High quality evidence is not available due to the rarity of the disease and the data are from only small case series. In our case the tumor was completely excised with radical cystectomy. Unfortunately the patient returned to his homeland 5 months after the operation and longer follow is not available.

\section{Conclusions}

Bladder SFTs are extremely rare mesenchymal neoplasms, usually arising from the bladder submucosa. Although they usually have benign features, their clinical behavior cannot be safely predicted. Signs and symptoms are non specific, while radiology findings are indictive of an enhancing bladder lesion.

Immunohistochemistry is pivotal for the diagnosis of SFTs. Although the literature is sparse and the level of evidence is low, radical surgical excision is the mainstay of treatment.

\section{REFERENCES}

1. Cheng SH, Wang SS, Lee CH, et al. Malignant solitary fibrous tumor of the urinary bladder. J Chin Med Assoc. 2012; 75:479-82.

2. Varaldo M, Ferrarazzo C, Tunesi $G$, et al. Solitary fibrous tumor. Solitary fibrous tumor. Rare Tumors. 2010; 2:e64.

3. Spairani C, Squillaci S, Pitino A, et al. A case of concomitant occurrence of solitary fibrous tumor and urothelial high-grade invasive carcinoma of the urinary bladder. Int J Surg Path. 2014; 22:252-9.

\section{Correspondence}

Zisis Kratiras, MD (Corresponding Author)

zkratiras@gmail.com

Diomidis Kozyrakis, MD

dkozirakis@yahoo.gr

Department of Urology, General Hospital of Volos

Dimokratias 126, 37300 Volos (Greece)

Vasileios Spapis, MD

vspapis@hotmail.com

Department of Urology, Hippokratio General Hospital of Athens, Athens (Greece)

Efthymios Koniaris, MD

ethimiok@hotmail.com

Department of Pathology, Hippokratio General Hospital of Athens, Athens (Greece)

Konstantinos Skriapas, MD

kostas.skriapas@hotmail.com

Department of Urology, General Hospital of Larisa, Larisa (Greece) 